\title{
Effect of microscopic diffusion on asteroseismic properties of intermediate-mass stars
}

\author{
J. Provost ${ }^{1}$, G. Berthomieu ${ }^{1}$, L. Bigot ${ }^{2}$, and P. Morel ${ }^{1}$ \\ 1 Département Cassiopée, UMR CNRS 6202, Observatoire de la Côte d'Azur, BP 4229, 06304 Nice Cedex 4, France \\ e-mail: Janine.Provost@obs-nice.fr \\ 2 Niels Bohr Institute, Juliane Maries Vej 30, 2100 Copenhagen, Denmark
}

Received 2 June 2004 / Accepted 31 October 2004

\begin{abstract}
Results on the effects of microscopic diffusion on the modeling and oscillations of solar-like stars are discussed. Comparisons between models with and without microscopic diffusion, subjected to observational constraints, namely effective temperature, luminosity, surface metallicity and mass derived from photometry, spectroscopy and astrometry, are presented. We point out and discuss a signature of microscopic diffusion on the oscillation frequencies.
\end{abstract}

Key words. stars: oscillations - stars: evolution - stars: oscillation - diffusion

\section{Introduction}

From recent ground-based asteroseismic observations, positive detections of oscillations similar to the five-minute solar oscillations have been made in a number of nearby stars, like $\alpha$ Cen, $\eta$ Boo, Procyon (e.g. Bouchy \& Carrier 2002; Kjeldsen et al. 2003; Martić et al. 2004). Future spatial missions hopefully will provide access to a large number of stars. These measurements will allow a better knowledge of the physics of the stellar interiors. In this work we study the effect of microscopic diffusion in the interpretation of seismic data of solar-like stars. This physical process changes the atmospheric abundances and removes some discrepancies between models and observations. In the solar case, seismic interpretation of the frequencies has shown the necessity of including microscopic diffusion in the modeling. The physical description of microscopic diffusion has been given by Burgers (1969). It has been included in stellar evolutionary codes in different ways (e.g. Cox et al. 1989; Proffitt \& Michaud 1991; Michaud \& Proffitt 1993; Thoul et al. 1994).

For stellar models of intermediate mass with mass larger than $1.4 M_{\odot}$ - in fact for any model without an extended external convective zone - the use of microscopic diffusion alone produces a depletion of helium and heavy elements and a concomitant enhancement of the hydrogen content at the surface. This strong depletion is not observed. Several factors acting against this large gravitational settling have been proposed. If one includes radiative accelerations (e.g. Alecian et al. 1989) and recalculates the local mean Rosseland opacity at each time step (e.g. Richer et al. 2000), this leads in some situations to an "iron" convective zone that inhibits the efficiency of the settling, but do not solve the general problem. Other ways involve in taking into account different processes, like mass loss
(Chaboyer et al. 1999), rotation induced mixing (e.g. Richard et al. 1996) or the use of an hypothetical mixed reservoir (Richer et al. 2000). As done by Heger et al. (2000) in their stellar evolutionary code, and according to the prescription of Morel \& Thévenin (2002), we use an additional mixing - see Sect. 2.

The main goal of this paper is to extract a signature of microscopic diffusion from the analysis of the theoretical oscillations of stellar models of intermediate mass. A model computed with microscopic diffusion is considered hereafter as target model. Then we compare its structure and oscillations to those of models computed without microscopic diffusion and with the same mass, effective temperature, luminosity and surface metallicity. These quantities have been chosen because they correspond to observational constraints which may be derived from astrometry, photometry and spectroscopy. Imposing luminosity and effective temperature corresponds to forcing a given radius. This quantity can be independently provided by VLTI measurements, as has been done for some stars like $\alpha$ Cen and Procyon (Kervella et al. 2003, 2004). Here the constraints have been chosen close to the Procyon A values, but with solar metallicity.

In a first step, we impose that these constraints are exactly satisfied for the "observable" parameters, namely effective temperature, luminosity and surface metallicity. By exactly we mean relative differences smaller than $10^{-3}$ for effective temperature and luminosity, and smaller than $10^{-2}$ for the surface metallicity. The way the models and oscillations are computed is described in Sect. 2. We study first the sensitivity of including in the modeling different formalisms for the description of the microscopic diffusion and we show that it does not significantly change the asteroseismic properties of the target model. 
Then we analyze the effect of diffusion on the different frequency combinations and we extract a signature of the microscopic diffusion on the oscillations by comparing models without diffusion with the target one (Sect. 3). In this comparison the physics of the models is fixed. However, the values of the parameters describing the convection are not known in the stellar case. We discuss in Sect. 4 the effect of slightly varying the modeling parameters, i.e. mixing length and core overshooting.

In a second step we take into account the fact that, for real stars, the observational constraints, i.e. the luminosity, the effective temperature and the surface metallicity that we use for modeling are measured with some uncertainties. In Sect. 5 we study the effect of varying slightly the observational constraints, within typical observed ranges. We try to determine if the signature of the microscopic diffusion on the oscillations remains relevant despite the uncertainties on these parameters.

\section{Models and oscillations}

\subsection{Physics of models}

All the models have been computed with the CESAM code (Morel 1997), with the following physics: nuclear data from the NACRE collaboration (e.g. Morel et al. 1999), the EFF equation of state (Eggleton et al. 1973), OPAL opacities extended with low temperatures opacities from Alexander \& Ferguson (1989) (routine kindly provided by Y. Lebreton), the Eddington law for the atmosphere description (see Morel et al. 1994), and starting with homogeneous ZAMS models. These models (cf. Kippenhahn \& Weigert 1991) are homogeneous stellar models computed with a given set of modeling parameters (initial mass, mixing length, initial abundances, core overshooting parameter,...) but without gravothermal energy release.

The convection is described according to Canuto \& Mazitelli (1991) with a mixing-length parameter $\lambda$ of order unity. Clear evidence for the presence of moderate core overshooting in stellar evolution has been pointed out by different authors to fit isochrones of open clusters and eclipsing binary data (e.g. Ribas et al. 2000). We use an overshooting of the convective core over the distance equal to $\zeta \min \left(H_{\mathrm{p}}, r_{\mathrm{c}}\right)$, where $H_{\mathrm{p}}$ and $r_{\mathrm{c}}$ are the pressure height scale and the radius of the convective core. We have chosen an often used value of the overshooting parameter $\zeta=0.2$.

The microscopic diffusion is taken into account according to either Michaud \& Proffitt (1993) or Burgers (1969). The calculations of collisional integrals and resistance coefficients are from Paquette et al. (1986) ${ }^{1}$.

In our case, i.e. a $1.5 M_{\odot}$ main sequence star, including radiative accelerations do not sufficiently limit the strong depletion of helium and heavy element content in the upper layers of the envelope. We follow in this paper the suggestion by Morel \& Thévenin (2002) to include an additional mixing, proportional to the radiative viscosity, parametrized by

\footnotetext{
${ }^{1}$ The detailed description can be found in "Notice de CESAM", available on request on the WEB site http://www.obs-nice.fr/morel/fichier_perso.html.
}

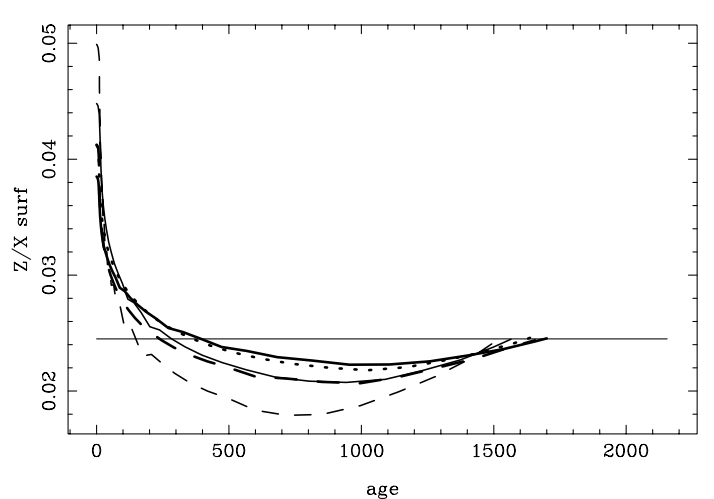

Fig. 1. Variation of the surface metallicity $(Z / X)_{\mathrm{S}}$ as a function of age: as a result of the microscopic diffusion, $(Z / X)_{S}$ varies along the stellar evolution for the models $\mathrm{D}_{0}$ (thick full), $\mathrm{D}_{1}$ (dotted), $\mathrm{D}_{2}$ (full), $\mathrm{D}_{3}$ (short dashed) and $\mathrm{D}_{4}$ (long dashed). The horizontal line shows the constant value of $(Z / X)_{S}$ for model $\mathrm{S}_{0}$.

$\mathcal{D}_{\mathrm{R}}$. In optically thick medium, the kinematic radiative viscosity is expressed as (e.g. Mihalas \& Weibel-Mihalas 1984, pp. 461-472):

$v_{\mathrm{rad}}=\frac{4}{15} \frac{a T^{4}}{c \kappa \rho^{2}}$,

where $a$ is the radiation density constant, $c$ is the speed of light in a vacuum and $\kappa$ is the mean Rosseland opacity. The radiative viscosity arises because the photons deposit their momentum in the fluid element into which they are absorbed (Mihalas \& Weibel-Mihalas 1984). Like the diffusion due to molecular collisions, the radiative diffusion due to radiative collisions has an isotropic component, the radiative diffusivity generating mixing associated with $v_{\text {rad }}$, and an anisotropic component causing the radiative accelerations that act against gravity (Alecian 1996). Figure 1 of Morel \& Thévenin (2002) shows examples of comparisons between molecular and radiative viscosities.

The radiative mixing and the mixing resulting from molecular collisions are independent processes. Therefore the molecular and the radiative diffusivities are additive processes. Contrary to the molecular case, there is no complete microscopic treatment of this radiative mixing. In Morel \& Thévenin (2002), it is described phenomenologically by a term proportional to the radiative viscosity and to a free parameter $\mathcal{D}_{\mathrm{R}}$. This parameter, calibrated to reproduce the Hyades abundances, is close to one. The use of this description of the radiative viscosity, although empiric, is an efficient additional mixing process (Heger et al. 2000; Morel \& Thévenin). As far as we know, it is the simplest recipe used in stellar evolutionary modeling to mimic some kind of turbulent transport to limit the not observed large gravitational settling.

All the models are obtained by adjusting the three quantities (the initial helium and heavy element contents and the age) to fit the luminosity, effective temperature and surface metallicity of the target model. As a result we obtain the helium abundance in the atmosphere and the structure of the models. 


\subsection{Asteroseismic analysis}

Intermediate-mass stars are solar-like stars, which means that the convective envelope is assumed to excite acoustic modes like in the Sun. As in the solar case we expect to derive the internal structure of the star from the oscillations, despite the fact that we can observe only low degree modes. Many observations, ground-based and spatial, have been devoted to this purpose. Here our goal is to test our capability to extract a signature of the microscopic diffusion from the oscillation frequencies. Thus we analyze this effect by computing for our models the adiabatic oscillation frequencies, $v_{n, \ell}$, for radial orders $n=5$ to 27 and for degrees $\ell=0$ to 3 . We directly compare these frequencies and the large and small spacings:

$\Delta v_{n, \ell}=v_{n, \ell}-v_{n-1, \ell}$,

$\delta v_{02}=v_{n+1, \ell=0}-v_{n, \ell=2}$

$\delta v_{01}=2 v_{n, \ell}-\left(v_{n, \ell+1}-v_{n-1, \ell+1}\right)$,

for models computed with various assumptions.

We recall that the large spacing $\Delta v_{n, \ell}$ is mainly sensitive to the outer layers. At high frequency (i.e. high radial order) this quantity becomes almost constant and proportional to $\left(\int \mathrm{d} r / c\right)^{-1}$. The sound speed $c, c \sim \sqrt{\mathcal{R} T / \mu}$, depends on temperature $T$ and mean molecular weight $\mu$. We also introduce the mean large spacing $\overline{\Delta v}$, obtained according to Berthomieu et al. (1993) with radial order from 17 to 25 (i.e. about 950 to $1450 \mu \mathrm{Hz}$ ). This quantity varies as $\sqrt{M / R^{3}}$. The small spacings $\delta v_{02}$ and $\delta v_{01}$ are very sensitive to the stellar core.

\section{Seismic signature of microscopic diffusion}

\subsection{Properties of target model; sensitivity to different microscopic diffusion formalisms}

A target model $\mathrm{D}_{0}$ is computed from a homogeneous ZAMS with $M=1.5 M_{\odot}, \log L / L_{\odot}=0.8475, T_{\text {eff }}=6506 \mathrm{~K}$, $(Z / X)_{\mathrm{S}}=0.0245$, using the simplified formalism of Michaud $\&$ Proffitt and $\mathcal{D}_{\mathrm{R}}=2$ (cf. Table 1). It is a main sequence model with a small convective core and a thin convective envelope. If we take into account the pre-main sequence phase in computing the target model, we obtain a model satisfying the constraints and which has the same structure properties as $\mathrm{D}_{0}$, in particular the size of convective zones. It has the same oscillation properties. Only the age is larger by about $10 \mathrm{Myr}$.

Figure 1 shows that, due to microscopic diffusion, the ratio between heavy element content and hydrogen content at the surface $(Z / X)_{\mathrm{S}}$ varies along the stellar evolution. From the ZAMS until about $1 \mathrm{Gyr}$, due to the gravitational settling, the surface abundances of helium and heavy elements decrease. After $1 \mathrm{Gyr}$, the density in the envelope decreases. This leads to an increase of the mixing modelled by $\mathcal{D}_{\mathrm{R}}$, with the consequence of a mild dredge-up which increases slightly the helium and metallicity at the surface.

Figure 2 shows the variation of the chemical composition along the radius. The large variation of $\mathrm{Z} / \mathrm{X}$ between the radiative interior and convective core is mainly due to the variation of the hydrogen content. For the models computed with
Table 1. Global characteristics of the target model $\mathrm{D}_{0}$ and models $\mathrm{D}_{i, i=1,4}$, computed with microscopic diffusion, either from Michaud \& Proffitt (MP) or Burgers (BR), and of the model S without diffusion. The meanings of symbols are: mixing parameter $\mathcal{D}_{\mathrm{R}}$, initial chemical composition $(Z / X)_{i}$ and $Y_{i}$, surface helium content $Y_{\mathrm{S}}$, age (in Myr), radius of the base of the external convection zone $r_{\mathrm{ZC}}$ and dimension of convective core $r_{\mathrm{c}}$ (in stellar radius unit), central hydrogen content $X_{\mathrm{c}} \cdot \overline{\Delta v}$ is the mean large spacing (in $\mu \mathrm{Hz}$ ). For all the models: $M / M_{\odot}=1.5, \log L / L_{\odot}=0.8475, T_{\text {eff }}=6506 \mathrm{~K},(Z / X)_{\mathrm{S}}=0.0245$, and core overshoot parameter $\zeta=0.2$.

\begin{tabular}{lllllll}
\hline \hline & $\mathrm{D}_{0}$ & $\mathrm{D}_{1}$ & $\mathrm{D}_{2}$ & $\mathrm{D}_{3}$ & $\mathrm{D}_{4}$ & $\mathrm{~S}_{0}$ \\
& $\mathrm{MP}$ & $\mathrm{MP}$ & $\mathrm{MP}$ & $\mathrm{BR}$ & $\mathrm{BR}$ & - \\
\hline $\mathcal{D}_{\mathrm{R}}$ & 2 & 1.5 & 1 & 1 & 2 & - \\
$\left(\frac{\mathrm{Z}}{X}\right)_{i}$ & 0.0385 & 0.0412 & 0.0448 & 0.0499 & 0.0413 & 0.0245 \\
$Y_{i}$ & 0.32 & 0.3272 & 0.3354 & 0.3456 & 0.3265 & 0.2728 \\
& & & & & & \\
$Y_{\mathrm{S}}$ & 0.208 & 0.200 & 0.189 & 0.201 & 0.217 & 0.2728 \\
$\mathrm{Age}$ & 1700 & 1643 & 1565 & 1515 & 1656 & 2133 \\
& & & & & & \\
$r_{\mathrm{ZC}}$ & 0.915 & 0.916 & 0.915 & 0.914 & 0.917 & 0.902 \\
$r_{\mathrm{c}}$ & 0.076 & 0.077 & 0.078 & 0.079 & 0.077 & 0.070 \\
$X_{\mathrm{c}}$ & 0.283 & 0.284 & 0.293 & 0.288 & 0.285 & 0.242 \\
$\overline{\Delta v}$ & 54.62 & 54.59 & 54.60 & 54.60 & 54.69 & 54.92 \\
\hline
\end{tabular}

diffusion, we see a region located below the outer convection zone, from 0.9 to 0.7 stellar radii, where the additional mixing smooths the transition between the convective zone and the inner radiative zone, where microscopic diffusion is at work.

To test the sensitivity of the target model to the description of diffusion, we computed models $\mathrm{D}_{i, i=1,4}$ with the simplified formalism of Michaud \& Proffitt and $\mathcal{D}_{\mathrm{R}}=1.5$ and 1 , and with the more complex formalism of Burgers and $\mathcal{D}_{\mathrm{R}}=2$ and 1 (cf. Table 1) and we compared their properties. These different models, calibrated in luminosity, effective temperature and surface metallicity, have slightly different ages, ranging from 1500 to 1700 Myr (Fig. 1 and Table 1). All the models have a convective core of the order of 0.1 stellar radius with different homogeneous chemical compositions (Fig. 2).

For all these models, the surface helium content changes by about $10 \%$. It is smaller with smaller $\mathcal{D}_{\mathrm{R}}$ which allows larger settling of helium and heavy elements towards the center. The surface helium content $Y_{\mathrm{S}}$ ranges from 0.19 to 0.22 . Thus models with diffusion require rather high initial helium $Y_{i}$ and heavy element $(Z / X)_{i}$ contents larger than the surface values by about a factor of 1.5 .

The difference of structure measured by the relative sound speed difference $\delta c / c$ plotted in Fig. $3 \mathrm{~b}$ is very small, of the order of $1 \%$. Thus the seismic properties of the models are expected to be very close. The frequency differences $\delta v$ between model $\mathrm{D}_{0}$ and the models $\mathrm{D}_{i, i=1,4}$ are given in Fig. $4 \mathrm{a}$. The values of $\delta v$ corresponding to p-modes are very small for $\mathrm{D}_{0}-\mathrm{D}_{i}$, except in the low frequency domain. In this domain some modes show a mixed character. They behave like a gravity mode in the core and an acoustic mode in the envelope. This is illustrated in Fig. 4b, which represents the contribution 


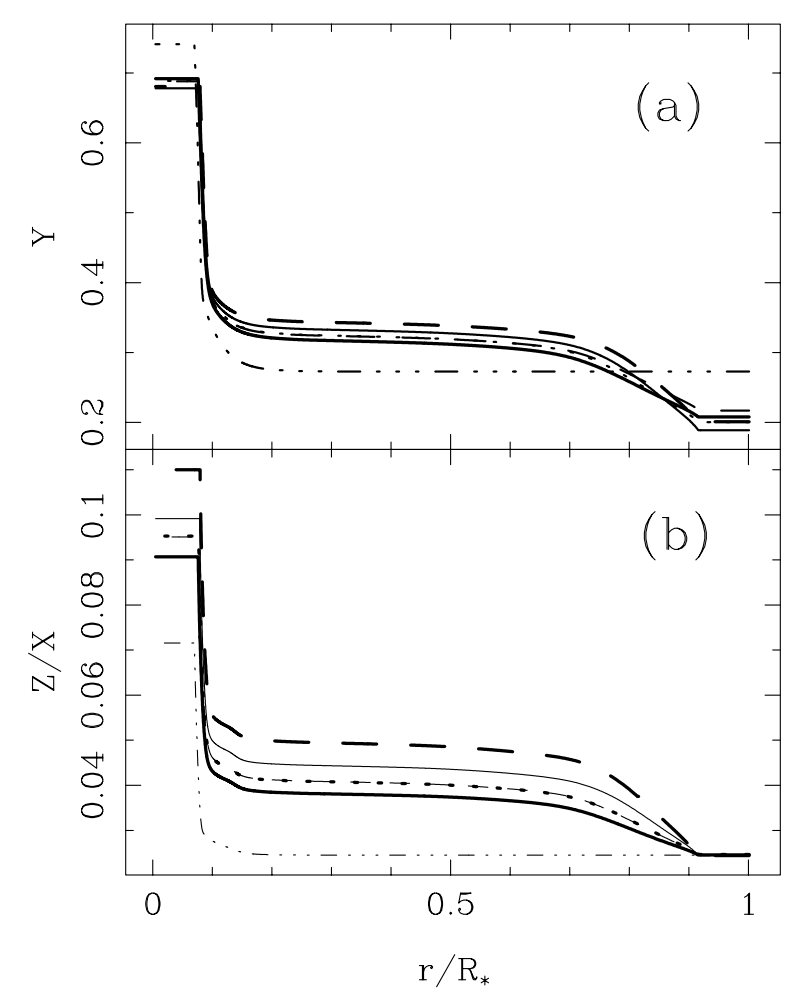

Fig. 2. Helium content $\mathrm{Y}$ a) and metallicity $\mathrm{Z} / \mathrm{X} \mathbf{b}$ ) as a function of the stellar radius, for the models $\mathrm{D}_{0}$ (thick full), $\mathrm{D}_{1}$ (dotted), $\mathrm{D}_{2}$ (full), $\mathrm{D}_{3}$ (long dashed), $\mathrm{D}_{4}$ (short dashed) and $\mathrm{S}_{0}$ (dash dot dot dot).

of the pressure perturbation to the frequency of the modes of model $\mathrm{S}_{0}$, defined below. This contribution is close to unity for p-modes, close to zero for pure gravity or surface modes and in between for mixed-modes. The differences $\delta v$ between $\mathrm{D}_{0}$ and the other models $\mathrm{D}_{i, i=1,4}$ are very small, less than $2 \mu \mathrm{Hz}$, in agreement with the fact that the values of the mean large spacings $\overline{\Delta v}$ given Table 1 are very close for these four models.

The large and small spacings plotted in Fig. 5 are very close for all the models $\mathrm{D}_{i}$. The core structure of the different models clearly visible in Fig. $3 b$ gives rise to changes of $0.5 \mu \mathrm{Hz}$ for $\delta v_{01}$ at a frequency of $1 \mathrm{mHz}$ (Fig. 4b).

Although the different descriptions of microscopic diffusion modify the helium content significantly, they do not much influence the asteroseismic properties of the calibrated models.

\subsection{Comparison between models with and without microscopic diffusion}

The effect of microscopic diffusion can be seen by comparing the properties of model $D_{0}$ and of model $S_{0}$ computed without diffusion, with the same luminosity, effective temperature and surface metallicity (Table 1 ). The model $\mathrm{D}_{0}$ has a larger initial helium but a much lower surface helium content relative to model $\mathrm{S}_{0}$. Its age is smaller by $400 \mathrm{My}$ and its convective core is larger by $7 \%$. This induces a central hydrogen content larger by $20 \%$. The depth of the external convective zone is smaller by $1.4 \%$ in the model $\mathrm{D}_{0}$ in agreement with the fact that the heavy element content at the surface $Z_{S}$ is smaller.

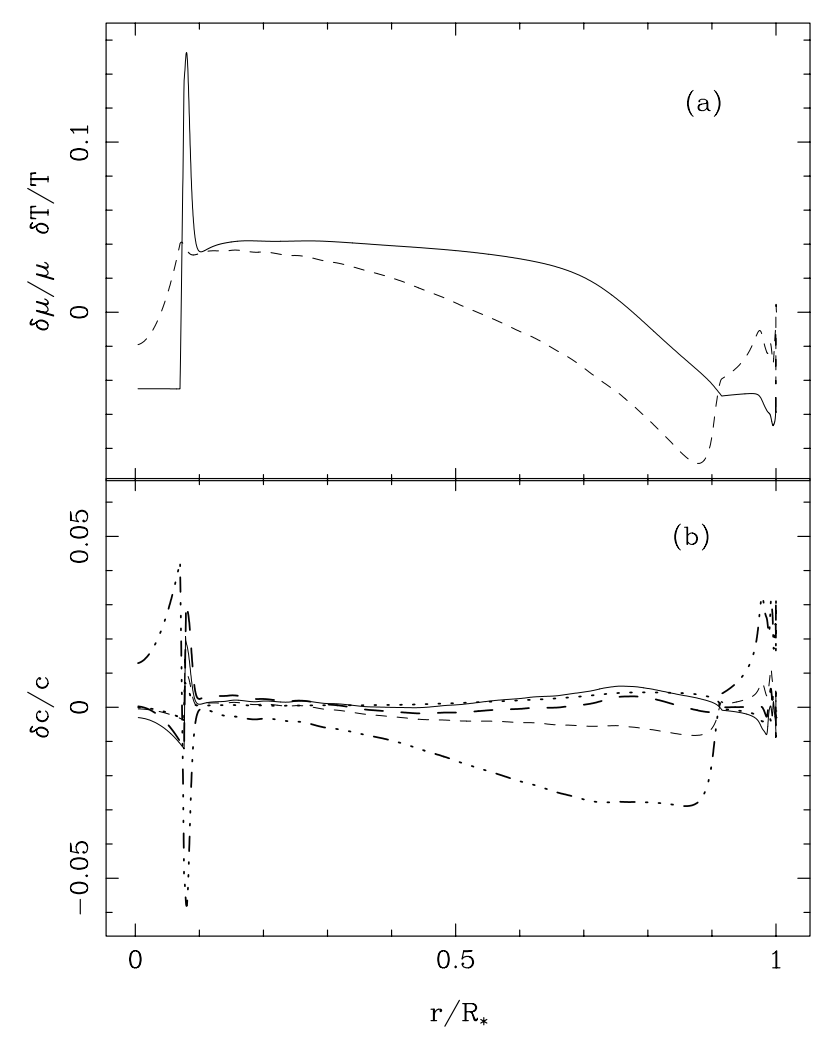

Fig. 3. a) Relative difference of temperature (dashed line) and mean molecular weight (full line) between models $D_{0}$ and and $S_{0}\left(D_{0}-S_{0}\right)$, as a function of the stellar radius. b) Relative difference of sound speed between models $\mathrm{D}_{0}$ and respectively $\mathrm{D}_{1}$ (dotted), $\mathrm{D}_{2}$ (full), $\mathrm{D}_{3}$ (long dashed), $\mathrm{D}_{4}$ (short dashed) and $\mathrm{S}_{0}$ (dash dot dot dot), as a function of the stellar radius.

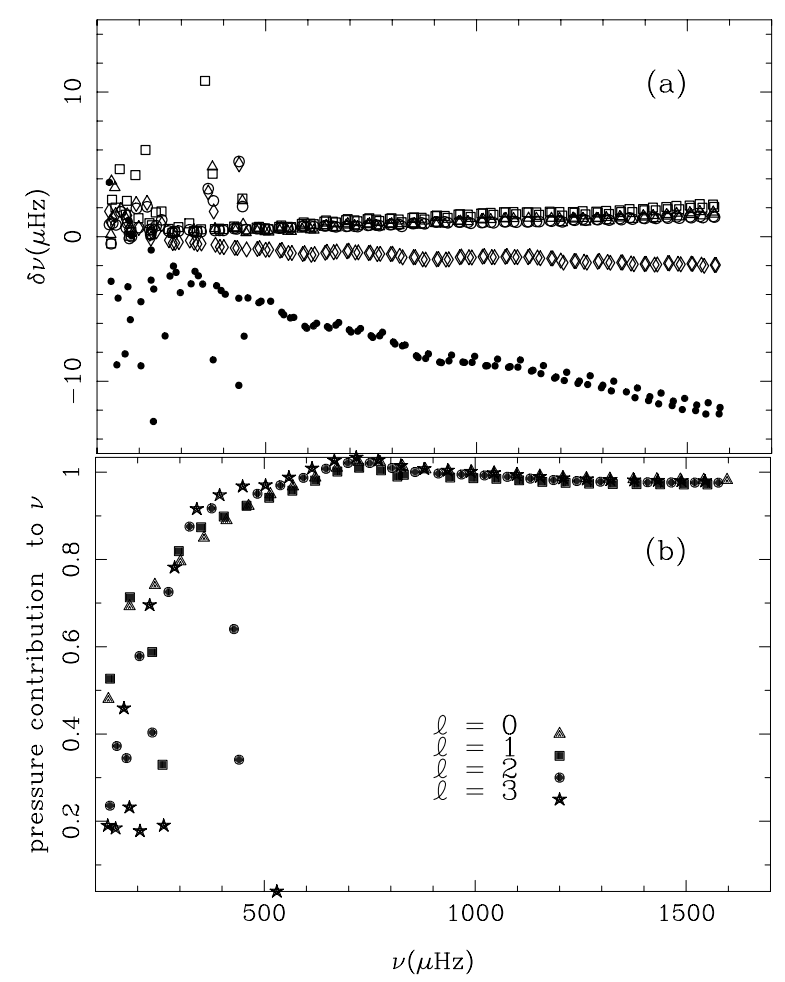

Fig. 4. a) Difference of frequencies between model $D_{0}$ and $D_{i, i=1,4}$ (open symbols) and $S_{0}$ (full circle). b) Contribution of the pressure perturbation to the frequencies of the oscillation modes of model $\mathrm{S}_{0}$. 


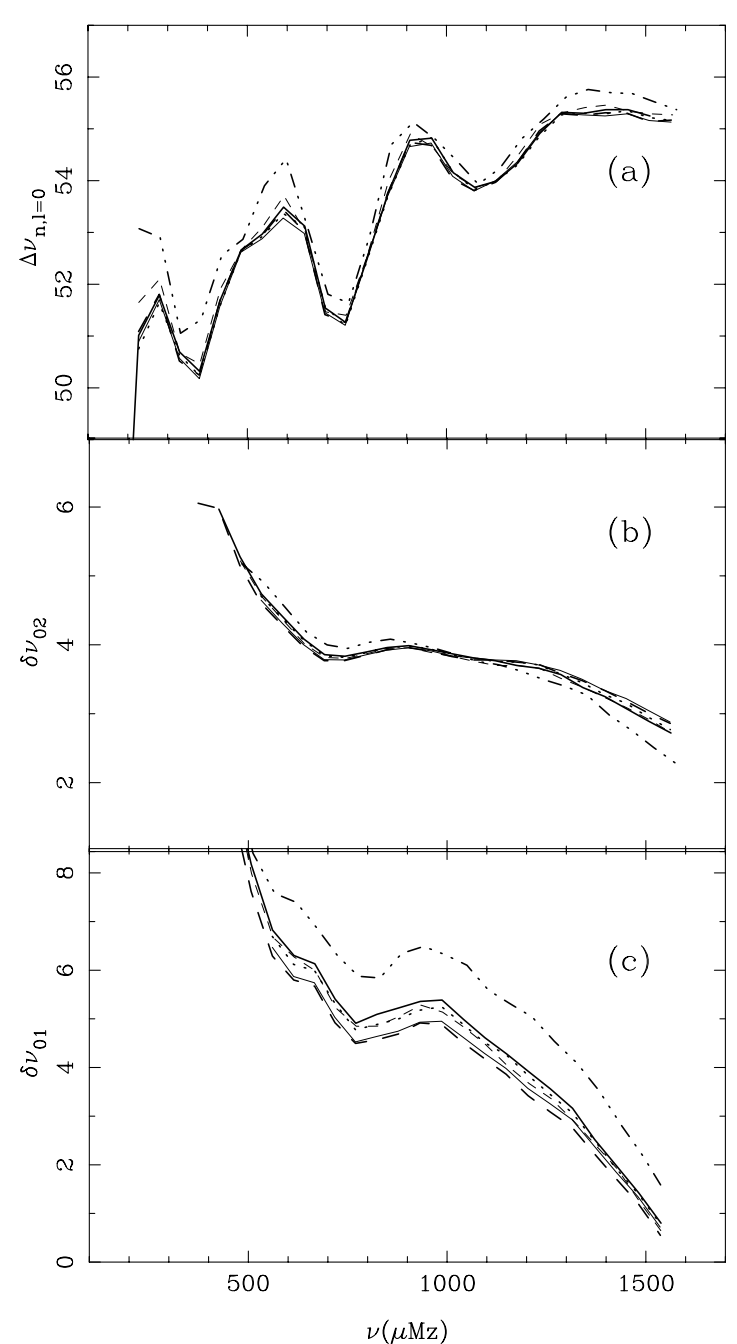

Fig. 5. Variation of the large spacings $\Delta v_{n, \ell=0}$ a) and the small spacings $\left.\delta v_{02} \mathbf{b}\right)$ and $\delta v_{01} \mathbf{c}$ ), as a function of the frequency for models computed with slightly different descriptions of the microscopic diffusion $\left(\mathrm{D}_{0}\right.$ (full thick), $\mathrm{D}_{1}$ (dotted), $\mathrm{D}_{2}$ (full), $\mathrm{D}_{3}$ (long dashed), $\mathrm{D}_{4}$ (short dashed) and for model $\mathrm{S}_{0}$ (dash dot dot dot)).

Figure $3 \mathrm{a}$ shows the variations of the relative differences of mean molecular weight $\delta \mu / \mu$ and of temperature $\delta T / T$ along the radius, for the models $\mathrm{D}_{0}$ and $\mathrm{S}_{0} . \delta \mu / \mu$ varies as the relative difference of the helium content in almost all the interior except in the ionisation helium and hydrogen zones. This induces sound speed differences $\delta c / c$ (Fig. 3b). The balance between a smaller temperature and a smaller amount of He both at surface and center, leading to a smaller mean molecular weight, results in a larger sound speed up to a few percent in the core and in the external convective layers for the model $D_{0}$. Beneath the convection zone, there is a minimum of $\delta c / c$ around 0.8 stellar radii, corresponding to a change of sign of $\delta \mu / \mu$ at the location where the helium content is almost the same in all models (see Fig. 2b).

As expected for models with the same mass and same radius, models $\mathrm{D}_{i, i=0,4}$ and $\mathrm{S}_{0}$ roughly satisfy the seismic constraint: they have a mean large frequency spacing $\overline{\Delta v}$ close to that of the target model $\mathrm{D}_{0}$. However, due to different chemical composition inducing a larger mean molecular weight for model $\mathrm{D}_{0}$, the sound speed is smaller for this model, hence $\overline{\Delta v}$ is smaller by $0.6 \%$. This results from a balance between positive and negative values of $\delta c / c$.

The microscopic diffusion induces a decrease of the frequencies, and consequently frequency differences $\delta v$ depending almost linearly on the frequency, down to $-10 \mu \mathrm{Hz}$ (Fig. 4a).

Figure 5a shows the variation of the large spacings $\Delta v_{n, \ell=0}$, as a function of frequency. At high frequency, these quantities have values close to the mean value $\overline{\Delta v}$ given in Table 1 . The large spacings are slightly higher for the model $\mathrm{S}_{0}$. The oscillatory behavior of $\overline{\Delta v}$ is due to the rapid variation of the adiabatic exponent $\Gamma$ in the HeII ionization zone (e.g. Gough 1991). The distance between the curves for models $D_{0}$ and $S_{0}$ reflects the different helium contents in the envelope of these models.

Figure $5 \mathrm{~b}$ shows that the small spacings $\delta v_{02}$ have almost the same values for all the models. They decrease with the frequency, but with a slightly smaller slope for all the models with diffusion. The values are the same around $1 \mathrm{mHz}$. The main result is that $\delta v_{01}$ is very sensitive to the effect of diffusion on the stellar structure and it has values lower by about $1.5 \mu \mathrm{Hz}$ for the models with diffusion, compared to the model $\mathrm{S}_{0}$ (Fig. 5c).

Thus, for a $1.5 M_{\odot}$ star in hydrogen burning phase, with given $T_{\text {eff }}, L / L_{\odot}$ and surface metallicity $(Z / X)_{S}$, models with microscopic diffusion have larger initial helium and heavy element contents $Y_{i}$ and $(Z / X)_{i}$, as a consequence of a larger convective core, a lower surface helium abundance $Y_{\mathrm{S}}$, and a smaller central helium $Y_{\mathrm{c}}$ resulting from a smaller age. We have found that the seismic properties are not modified much, except for a significant decrease of the small spacing $\delta v_{01}$, which constitutes the main signature of microscopic diffusion on oscillations.

\section{Effect of changing the convection parameters}

In this section we examine the effect of calibrating models similar to model $\mathrm{S}_{0}$, with the constraints of the target model, and varying the mixing-length and core overshoot parameters. The asteroseismic properties of models $\mathrm{S}_{i, i=1,4}$ (see Tables 2 and 3) are compared to those of the target model $\mathrm{D}_{0}$.

\subsection{Mixing-length}

We have changed the efficiency of the convection described according Canuto \& Mazitelli (1991) by varying the mixing length parameter around $\lambda=1$. A change of $\lambda$ will change principally the external part of the star, i.e. the radius of the base of the external convection zone. For decreasing $\lambda$, the models $S_{i}$ have an increasing radius of the external convection zone (Table 2). A decrease of $\lambda$ by $5 \%$ brings this radius and consequently the mean large spacing $\overline{\Delta v}$ close to the one of model $\mathrm{D}_{0}$. On the contrary, the age, helium content and convective core radius are not changed much. The age becomes smaller, but still larger than that of the target model.

These conclusions are also illustrated Fig. 6. There is a shift in frequency for the large spacing $\Delta v$ corresponding to the models with different $\lambda$ (Fig. 6a). For the three models $S_{i, i=0,1,2}$, the variation of $\delta v_{01}$ with frequency has the same behavior, clearly 
Table 2. Effect of changing the mixing-length parameter $\lambda$.

\begin{tabular}{lllll}
\hline \hline Model & $\mathrm{D}_{0}$ & $\mathrm{~S}_{1}$ & $\mathrm{~S}_{0}$ & $\mathrm{~S}_{2}$ \\
$\lambda$ & 1. & 1.05 & 1.0 & 0.95 \\
\hline & & & & \\
$(Z / X)_{i}$ & 0.0385 & 0.0245 & 0.0245 & 0.0245 \\
$Y_{i}$ & 0.32 & 0.272 & 0.273 & 0.274 \\
& & & & \\
$Y_{\mathrm{S}}$ & 0.208 & 0.272 & 0.273 & 0.274 \\
Age $(\mathrm{Myr})$ & 1700 & 2205 & 2133 & 2105 \\
& & & & \\
$r_{\mathrm{ZC}}$ & 0.915 & 0.891 & 0.902 & 0.917 \\
$r_{\text {core }}$ & 0.076 & 0.0684 & 0.070 & 0.0705 \\
$X_{\mathrm{c}}$ & 0.283 & 0.219 & 0.242 & 0.254 \\
$\overline{\Delta v}(\mu \mathrm{Hz})$ & 54.62 & 55.04 & 54.93 & 54.65 \\
\hline
\end{tabular}

Table 3. Effect of changing the core overshoot parameter $\zeta$.

\begin{tabular}{llllll}
\hline \hline Model & $\mathrm{D}_{0}$ & $\mathrm{D}_{5}$ & $\mathrm{~S}_{0}$ & $\mathrm{~S}_{3}$ & $\mathrm{~S}_{4}$ \\
$\zeta$ & 0.2 & 0.1 & 0.2 & 0.15 & 0.1 \\
\hline & & & & & \\
$(Z / X)_{i}$ & 0.0385 & 0.0379 & 0.0245 & 0.0245 & 0.0245 \\
$Y_{i}$ & 0.32 & 0.3246 & 0.273 & 0.276 & 0.280 \\
& & & & & \\
$Y_{\mathrm{S}}$ & 0.208 & 0.216 & 0.273 & 0.276 & 0.280 \\
Age $(\mathrm{Myr})$ & 1700 & 1580 & 2133 & 2055 & 1958 \\
& & & & & \\
$r_{\mathrm{ZC}}$ & 0.915 & 0.914 & 0.902 & 0.904 & 0.904 \\
$r_{\mathrm{c}}$ & 0.076 & 0.068 & 0.070 & 0.065 & 0.060 \\
$X_{\mathrm{c}}$ & 0.283 & 0.232 & 0.242 & 0.200 & 0.161 \\
$\overline{\Delta v}(\mu \mathrm{Hz})$ & 54.62 & 54.59 & 54.92 & 54.76 & 54.85 \\
\hline
\end{tabular}

different to that of model $\mathrm{D}_{0}$ (Fig. 6c). Except for $\delta v_{01}$, a small decrease of $\lambda$ gives a model $\mathrm{S}_{2}$ with seismic properties closer to those of model $\mathrm{D}_{0}$.

\subsection{Core overshoot parameter}

Decreasing the core overshoot parameter $\zeta$ mainly modifies the core properties, i.e. the size of the mixed core and consequently the central hydrogen content are smaller (Table 3). The structure of the stellar envelope is not much modified (Fig. 7a), so there is no effect on the large spacing. There is a slight change for the small spacing $\delta v_{02}$ (Fig. 7b). The main effect on frequency is to modify $\delta v_{01}$ (Fig. 7c). At high frequency, a decrease of $\zeta$ induces an increase of the value of $\delta v_{01}$ and a decrease of the slope. Thus the high frequency range does not allow one to discriminate between diffusion and core overshoot effect. This corresponds to the fact that the model $D_{5}$ with $\zeta=0.1$ has a core dimension close to that of the model $\mathrm{S}_{0}$ with $\zeta=0.2$. The asteroseismic signature of the diffusion remains in the intermediate frequency range below $1 \mathrm{mHz}$.

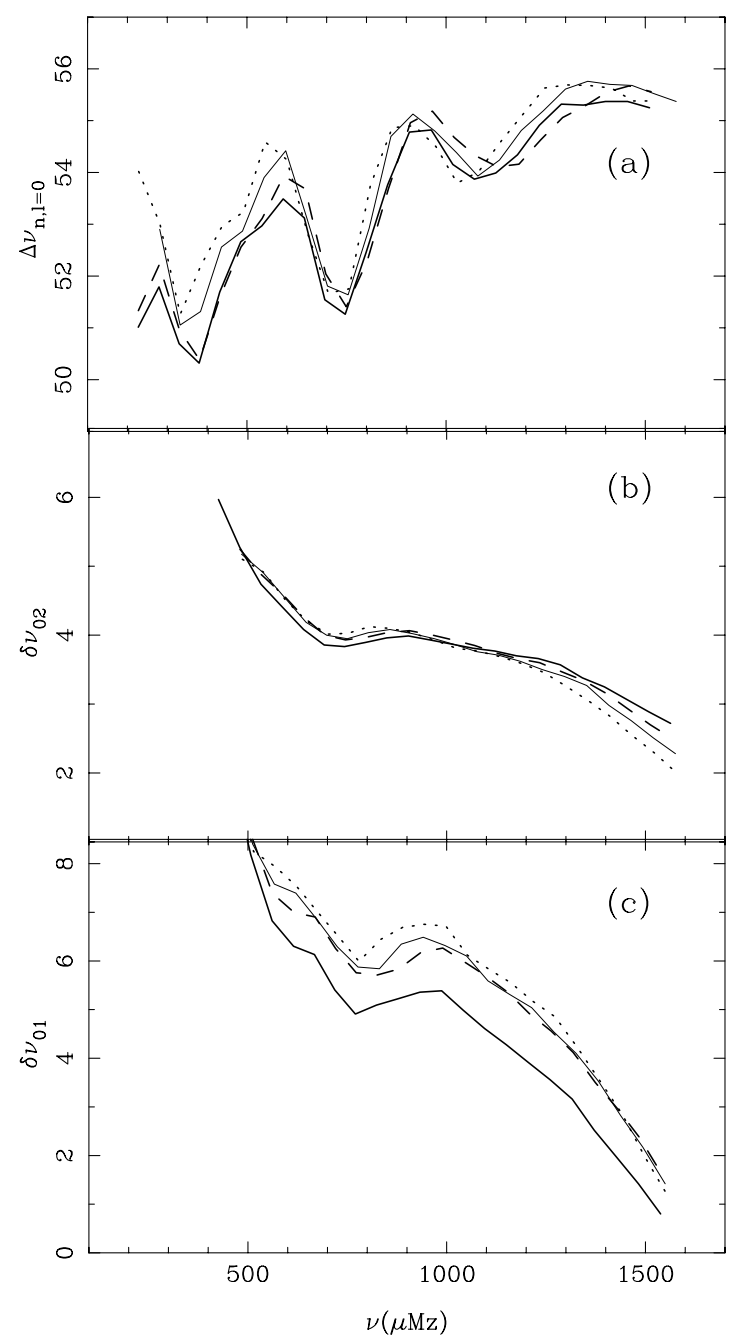

Fig. 6. Effect of changing the mixing-length parameter $\lambda$ : variation of the large and small spacings, respectively a)-c), as a function of the frequency, for model $\mathrm{D}_{0}$ (heavy full line) and models $\mathrm{S}_{1}(\lambda=1.05$, dotted $), S_{0}(\lambda=1.0$, full $), S_{2}(\lambda=0.95$, dashed $)$.

\section{Effect of the observational uncertainties}

In this section we examine if the signature on the oscillations of the microscopic diffusion remains if we relax the observational constraints, surface metallicity, mass and HR constraints, within typical observed ranges. In order to restrain the possible range of variation of these quantities, we assume that the oscillations of the model $\mathrm{D}_{0}$ are known, at least for the large separation $\Delta v$. Indeed the constraints of luminosity, effective temperature, mass and mean large frequency spacing are not independent. First we vary only the surface metallicity and in a second step we vary the mass and the HR constraints. In this section we keep the description of the convection fixed at $\lambda=1$.

\subsection{Change in surface metallicity}

We have computed calibrated models $S_{5,6}$, with the same mass, luminosity and effective temperature and hence the same radius as model $\mathrm{D}_{0}$, varying the surface metallicity from 0.0217 to 0.0270 (Table 4). The models have an age larger by $100 \mathrm{Myr}$ for 

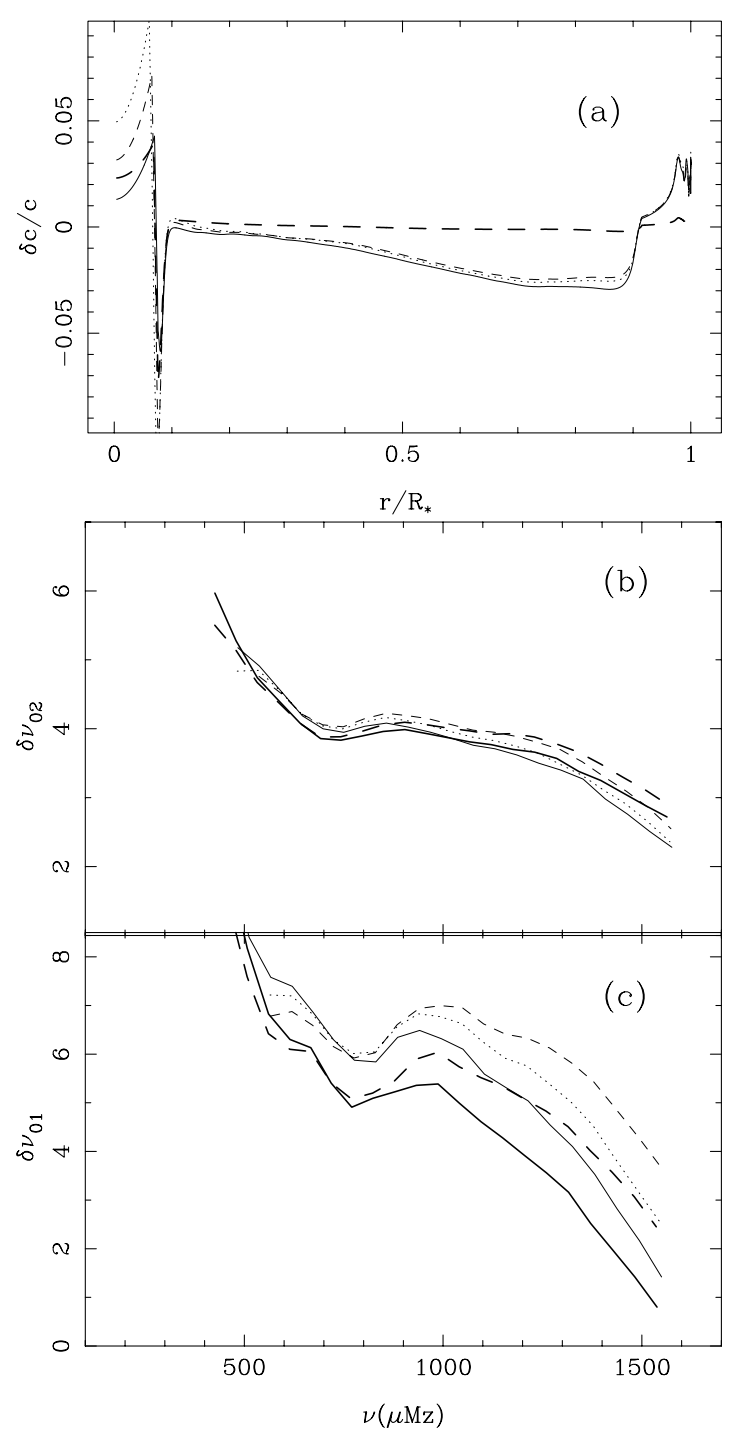

Fig. 7. Effect of changing the core overshoot parameter $\zeta$. a) Relative difference of sound speed between model $\mathrm{D}_{0}$ and models with different values of $\zeta$ core, respectively $\mathrm{S}_{0}\left(\zeta=0.2\right.$, full), $\mathrm{S}_{4}(\zeta=0.1$, dashed $)$ and $S_{3}(\zeta=0.15$, dotted), as a function of the stellar radius. b) and c) Small spacings $\delta v_{01}$ and $\delta v_{02}$ for $\mathrm{D}_{0}\left(\zeta=0.2\right.$ heavy full), $\mathrm{D}_{5}(\zeta=0.1$ heavy dashed) and the models $\mathrm{S}_{0}\left(\zeta=0.2\right.$, full), $\mathrm{S}_{4}(\zeta=0.1$, dashed $)$ and $S_{3}(\zeta=0.15$, dotted $)$.

a decrease of metallicity from 0.0245 to 0.0217 and their internal structure is almost unchanged, leading to a small effect on the oscillations. The large and small spacings, $\Delta v$ and $\delta v_{02}$, vary very slightly. The values of $\delta v_{01}$ are slightly larger for smaller metallicity. They are larger than the values for the model $D_{0}$ (Fig. 8). Thus the effect of microscopic diffusion cannot be compensated for by a variation of the metallicity alone.

\subsection{Changes in mass and in HR constraints}

We have computed models $S_{i, i=7,16}$ (Table 5) with mean large spacing close to that of model $\mathrm{D}_{0}$ keeping the constraints around those of $\mathrm{D}_{0}$ within realistic stellar uncertainty ranges, i.e. the mass $\pm 2 \%$, uncertainty which could be expected from GAIA data for binary stars, the surface metallicity around the

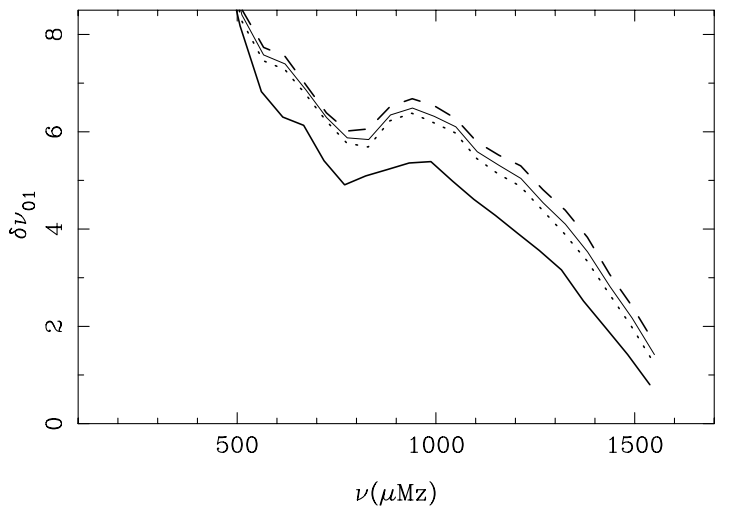

Fig. 8. Effect of changing the surface metallicity. Small spacing $\delta v_{01}$ for $\mathrm{D}_{0}$ (heavy full line) and the models of $\mathrm{S}_{0}(Z / X=0.0245$, full line), $\mathrm{S}_{5}(Z / X=0.0217$, dashed $)$ and $\mathrm{S}_{6}(Z / X=0.0270$, dotted $)$.

Table 4. Effect of changing the surface metallicity $(Z / X)_{\mathrm{S}}$.

\begin{tabular}{lllll}
\hline \hline Model & $\mathrm{D}_{0}$ & $\mathrm{~S}_{0}$ & $\mathrm{~S}_{5}$ & $\mathrm{~S}_{6}$ \\
$(Z / X)_{\mathrm{S}}$ & 0.0245 & 0.0245 & 0.0217 & 0.0270 \\
\hline & & & & \\
$(Z / X)_{i}$ & 0.0385 & 0.0245 & 0.0217 & 0.0270 \\
$Y_{i}$ & 0.32 & 0.2728 & 0.260 & 0.283 \\
& & & & \\
$Y_{\mathrm{S}}$ & 0.208 & 0.2728 & 0.2601 & 0.283 \\
Age $(\mathrm{Myr})$ & 1700 & 2133 & 2233 & 2058 \\
& & & & \\
$r_{\mathrm{ZC}}$ & 0.915 & 0.902 & 0.902 & 0.9021 \\
$r_{\mathrm{c}}$ & 0.076 & 0.070 & 0.068 & 0.71 \\
$X_{\mathrm{c}}$ & 0.283 & 0.242 & 0.236 & 0.244 \\
$\overline{\Delta v}(\mu \mathrm{Hz})$ & 54.62 & 54.92 & 54.93 & 54.82 \\
\hline
\end{tabular}

classical solar value, from 0.0217 to 0.0270 , and the effective temperature $\pm 50 \mathrm{~K}, \log L / L_{\odot} \pm 0.03$ and $\overline{\Delta v} \pm 0.2 \mu \mathrm{Hz}$. For a given mass and surface metallicity, the constraints on the large spacing lead to a constraint on the radius of the star roughly according to:

$\frac{\delta \overline{\Delta v}}{\overline{\Delta v}} \sim 3 / 2 \frac{\delta R}{R}$.

This restrains the area of the HR diagram where the calibrated model is located. These areas are given in Fig. 9 for $M / M_{\odot}=1.47$ and 1.53 and $(Z / X)_{\mathrm{S}}=0.0245$. To obtain these calibrated models, one varies the initial helium content within two extreme values corresponding to the extremities of each area, as in Fig. 9. Independently, the radius may be constrained by VLTI observations (Kervella et al. 2004). These extreme values are given in Fig. 10 for two metallicities, as a function of the mass of the models. Table 5 presents the global characteristics of some examples of such models calibrated in $\overline{\Delta v}$. For given $Y_{i},(Z / X)_{i}$ and mass, the ages of these models are in a range determined by the uncertainty assumed for $\overline{\Delta v}$. This range is about $30 \mathrm{Myr}$ corresponding to the range of uncertainty of $0.4 \mu \mathrm{Hz}$ we adopted for $\overline{\Delta v}$, as seen in the two first columns of Table 5. The two following columns correspond to models with mass $1.47 M_{\odot}$, surface metallicity $Z / X_{S}$ and the two 
Table 5. Effect of a change in observed constraints on the global characteristics of some models, with the values close to those of the target model: $M / M_{\odot}=1.5 \pm 2 \%,(Z / X)_{\mathrm{S}}=0.0245_{-0.028}^{+0.025}, T_{\text {eff }} \pm 50 \mathrm{~K}, \log L / L_{\odot} \pm 0.03$, and $\overline{\Delta v}=54.62 \pm 0.2 \mu \mathrm{Hz}$.

\begin{tabular}{|c|c|c|c|c|c|c|c|c|c|c|}
\hline \multirow[t]{2}{*}{ Model } & $\overline{\mathrm{S}_{7}}$ & $\overline{S_{8}}$ & $\mathrm{~S}_{9}$ & $\overline{S_{10}}$ & $\overline{S_{11}}$ & $\mathrm{~S}_{12}$ & $\overline{S_{13}}$ & $\overline{\mathrm{S}_{14}}$ & $\overline{S_{15}}$ & $\mathrm{~S}_{16}$ \\
\hline & \multicolumn{2}{|c|}{$\begin{array}{l}M / M_{\odot}=1.50 \\
(Z / X)_{i}=0.0245\end{array}$} & \multicolumn{2}{|c|}{$\begin{array}{l}M / M_{\odot}=1.47 \\
(Z / X)_{i}=0.0270\end{array}$} & \multicolumn{2}{|c|}{$\begin{array}{l}M / M_{\odot}=1.47 \\
(Z / X)_{i}=0.0217\end{array}$} & \multicolumn{2}{|c|}{$\begin{array}{l}M / M_{\odot}=1.53 \\
(Z / X)_{i}=0.0270\end{array}$} & \multicolumn{2}{|c|}{$\begin{array}{l}M / M_{\odot}=1.53 \\
(Z / X)_{i}=0.0217\end{array}$} \\
\hline$\overline{Y_{i}}$ & 0.270 & 0.270 & 0.299 & 0.290 & 0.278 & 0.269 & 0.277 & 0.267 & 0.254 & 0.244 \\
\hline Age (Myr) & 2190 & 2220 & 1971 & 2160 & 2130 & 2320 & 1955 & 2161 & 2130 & 2342 \\
\hline $\log L / L_{\odot}$ & 0.8421 & 0.8425 & 0.8516 & 0.8370 & 0.8554 & 0.8360 & 0.8657 & 0.8444 & 0.8658 & 0.8447 \\
\hline$T_{\text {eff }}$ & 6481 & 6459 & 6545 & 6453 & 6545 & 6456 & 6557 & 6457 & 6555 & 6456 \\
\hline$R / R_{\odot}$ & 2.093 & 2.111 & 2.077 & 2.089 & 2.086 & 2.096 & 2.103 & 2.112 & 2.105 & 2.118 \\
\hline$r_{\mathrm{ZC}}$ & 0.896 & 0.891 & 0.909 & 0.897 & 0.909 & 0.887 & 0.917 & 0.893 & 0.916 & 0.893 \\
\hline$r_{\mathrm{c}}$ & 0.069 & 0.068 & 0.071 & 0.069 & 0.068 & 0.066 & 0.072 & 0.070 & 0.070 & 0.068 \\
\hline$X_{\mathrm{c}}$ & 0.236 & 0.226 & 0.237 & 0.217 & 0.226 & 0.206 & 0.267 & 0.246 & 0.259 & 0.237 \\
\hline$\overline{\Delta v}$ & 54.81 & 54.45 & 54.72 & 54.77 & 54.46 & 54.55 & 54.67 & 54.67 & 54.67 & 54.73 \\
\hline
\end{tabular}

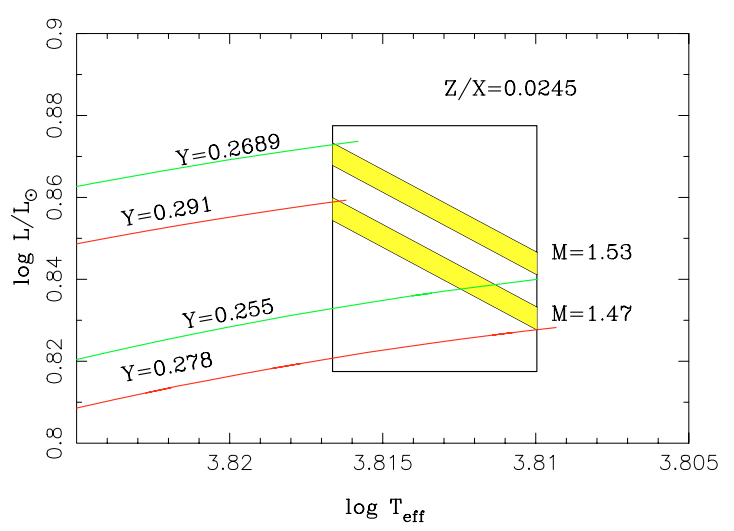

Fig. 9. The shaded areas correspond to the location, in the HR diagram, of calibrated models with $(Z / X)_{\mathrm{S}}=0.0245$, and two values of the mass: $M=1.47$ or 1.53 and $\log L / L_{\odot}, T_{\text {eff }}$ and $\overline{\Delta v}$ with the constraints defined in Sect. 4.2. The evolutionary tracks of models close to the border lines of these areas are labelled by their initial helium content $Y_{i}$. This is the way we define the range of $Y_{i}$ plotted in Fig. 10.

extreme values of initial helium content $Y_{i}$, as defined above. The difference of ages of these models, about $200 \mathrm{Myr}$, corresponds roughly to the crossing time of the HR diagram uncertainty box. The next columns show that for models of different mass and metallicity, relative to the target model $\mathrm{D}_{0}$, the helium content $\left(Y_{i}=Y_{\mathrm{S}}\right)$ is smaller than the initial helium content, but larger than the surface helium content of model $\mathrm{D}_{0}$. The ages are larger, by at least $300 \mathrm{Myr}$, and the central hydrogen $X_{\mathrm{c}}$ is smaller.

For all these models satisfying the above constraints, the large and small spacings $\Delta v, \delta v_{01}$ and $\delta v_{02}$ are represented in Fig. 11 and compared to the same quantities for the target model. The dispersion of the curves representing $\Delta v$, indicated by a grey strip in Fig. 11a, is a consequence of the different ages and helium contents of the models. As a result the signature of the diffusion is only clearly visible on the small spacing $\delta v_{01}$. In Fig. $11 \mathrm{c}$ the curve closest to that of the target model is obtained for the larger mass, metallicity and initial helium content, while the curve with the highest values is obtained for the smallest mass, metallicity and initial helium. These conclusions are valid for a given description of the convection.

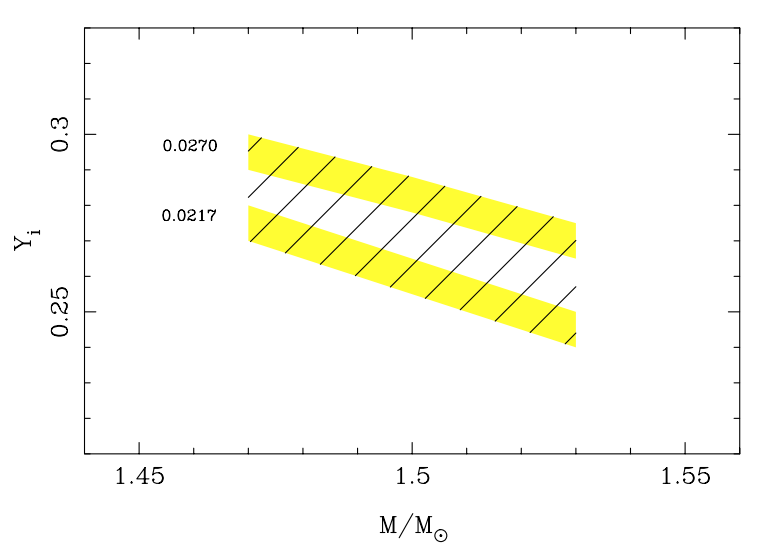

Fig. 10. Range of $Y_{i}$ and mass at given surface metallicity for models calibrated in $\overline{\Delta v}$ for the value of the target model $\mathrm{D}_{0}$. The two grey areas correspond respectively to $(Z / X)_{\mathrm{S}}=0.0270$ and 0.0217 . The hatched area corresponds to the full range of possible initial helium, as a function of the mass.

As seen in Sect. 3.2, different values of the core overshoot parameter for the target model and $S_{i}$ models lead to a mixing of the curves representing $\delta v_{01}$ in the high frequency range.

\section{Discussion and conclusion}

We have considered a target model for a $1.5 M_{\odot}$ star in the hydrogen burning phase taking into account microscopic diffusion. To analyse the effect of this diffusion on solar-like oscillations, we have compared the structure and oscillations of this target model to models without microscopic diffusion. This has been done first with the following assumed constraints, i.e. given effective temperature, luminosity and surface metallicity and keeping the physics fixed. We have found that the microscopic diffusion induces the following effects on the models: larger initial helium and heavy element contents, lower surface helium abundance, smaller age and consequently a larger central hydrogen content. Concerning the oscillations, we found a significant decrease of the small spacing $\delta v_{01}$. This constitutes an important signature of microscopic diffusion on oscillations.

We have studied the sensitivity of these results to the uncertainties of the modeling parameters and to the description of the 


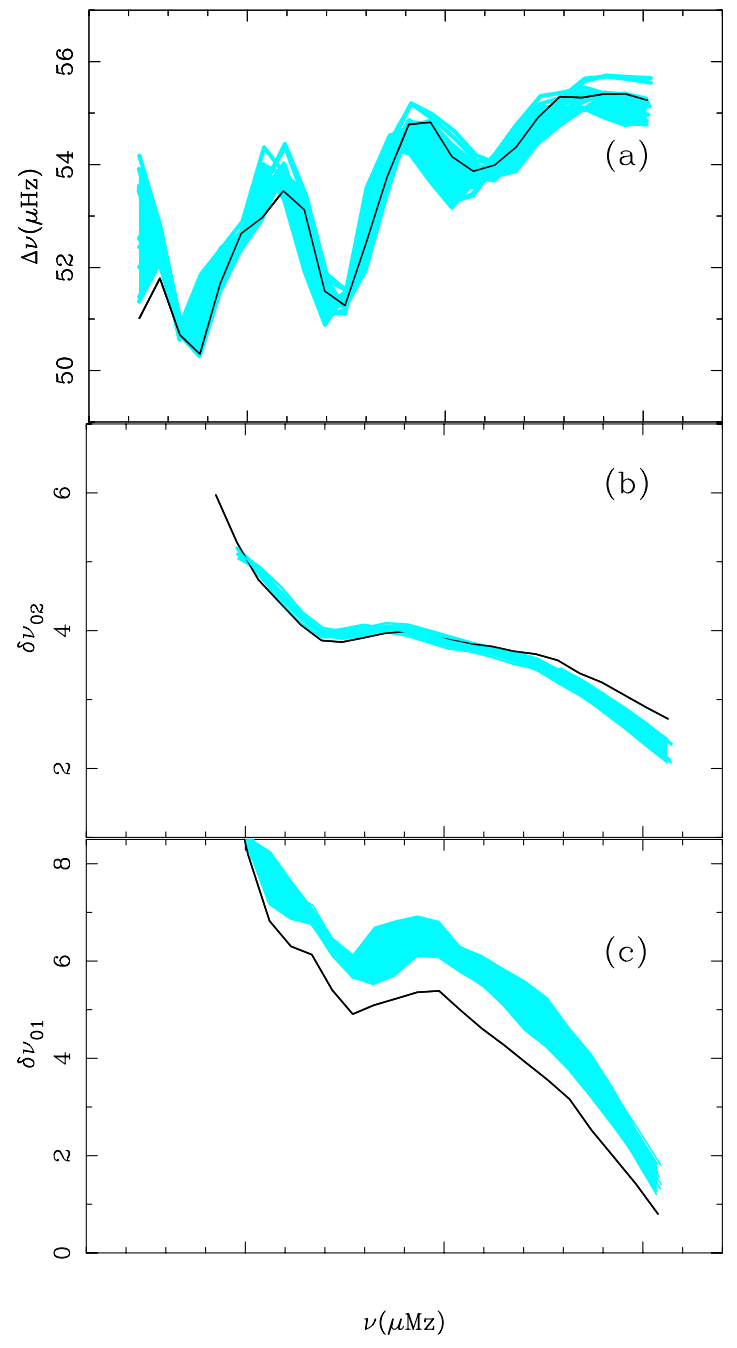

Fig. 11. Effect of changing the mass and HR constraints for models $\mathrm{S}_{i, i=7,16}$, calibrated to the target model value of $\overline{\Delta v}$, on the large a) and small spacings b) and c) (grey area), and compared to target model $\mathrm{D}_{0}$ (full line).

physics. We found that if we vary the constraints adopted, i.e. effective temperature, luminosity, surface metallicity and mass, close to the values of the target model within realistic uncertainty ranges, and keeping the mean large frequency spacing close to the value of $\mathrm{D}_{0}(54.62 \pm 0.2 \mu \mathrm{Hz})$, then the signature of microscopic diffusion on the small splitting $\delta v_{01}$ still remains. The effect of microscopic diffusion and of core overshooting can be disentangled only at intermediate frequency, below $1 \mathrm{mHz}$. We have also studied the sensitivity of our results to the description of the microscopic diffusion, using either the Michaud \& Profitt (1993) or Burgers (1969) descriptions, and varying the additional mixing parameter introduced by Morel \& Thévenin (2002). We have found that these slightly different descriptions of the diffusion provide almost the same global structure along the radius of the models and do not change at all the asteroseismic signature of the microscopic diffusion.
As a consequence, in order to be able to reproduce and interpret the oscillations of future targets of asteroseismic missions like COROT and to increase our understanding of the physics of the stellar interior of solar-like stars, one should consider microscopic diffusion in stellar modeling.

Acknowledgements. We are grateful to F. Thévenin for many valuable discussions. We thank the referee for constructive remarks and suggestions. This work has been performed using the computing facilities provided by the OCA program "Simulations interactives et Visualisation en Astronomie et Mécanique" (SIVAM). L.B. thanks EU for a Marie Curie fellowship.

\section{References}

Alecian, G., Michaud, G., \& Tully, J. 1989, A\&A, 411, 882

Alexander, D. R., \& Ferguson, J. W. 1994, ApJ, 437, 879

Berthomieu, G., Provost, J., Morel, P., \& Lebreton, Y. 1993, A\&A, 268,775

Bouchy, F., \& Carrier, F. 2002, A\&A 390, 205

Burgers, J. M. 1969, Flow equations for composite gases (New York and London: Academic Press)

Canuto, V. M., \& Mazitelli, I. 1991, ApJ, 370, 295

Chaboyer, B., Demarque, P., \& Guenther, D. B. 1999, ApJ, 525, L41

Cox, A. N., Guzik, J. A., \& Kidman, R. B. 1989, ApJ, 342, 1187

Eggleton, P. P., Faulkner, J., \& Flannery, B. P. 1973, A\&A, 23, 325

Gough, D. O. 1991, Comments on Helioseismic Inference, ed. Y. Osaki, \& H. Shibahashi Progress of Seismology of the Sun and Stars (Springer Verlag), 283

Heger, A., Langer, N., \& Woosley, S. E. 2000, ApJ, 528, 338

Kervella, P., Thévenin, F., Ségransan, D., et al. 2003, A\&A, 413, 251

Kervella, P., Thévenin, F., Morel, P., et al. 2004, A\&A, 413, 251

Kippenhahn, R., \& Weigert, A., Stellar structure and evolution (Springer Verlag)

Kjeldsen, H., Bedding, T. R., Baldry, I. K., et al. 2003, ApJ, 126, 1483

Martić, M., Lebrun, J. C., Appourchaux, T., \& Korzennik, S. 2004, A\&A, 418, 295

Michaud, G., \& Proffitt, C. R. 1993, Particule Transport Process, ed. A. Baglin, \& W. W. Weiss, Inside the Stars, IAU Coll., 137, ASP Conf. Ser., 40, 246

Mihalas, D., \& Weibel-Mihalas, B. 1984, Foundations of Radiation Hydrodynamics (Oxford University Press), 461

Morel, P., van't Veer, C., Provost, J., et al. 1994, A\&A, 286, 91

Morel, P. 1997, A\&AS, 124, 597

Morel, P., Pichon, B., Provost, J., \& Berthomieu, G. 1999, A\&A, 350, 275

Morel, P., \& Thévenin, F. 2002, A\&A, 390, 611

Paquette, C., Pelletier, C., Fontaine, G., \& Michaud, G. 1986, ApJS, 61,177

Proffitt, C. R. , \& Michaud, G. 1991, ApJ, 371, 584

Ribas, I., Jordi, C., \& Gimenéz, A. 2000, MNRAS, 318, L55

Richard, O., Vauclair, S., Charbonnel, C., \& Dziembowski, D. A. 1996, A\&A, 312, 1000

Richer, J., Michaud, G., \& Turcotte, S. 2000, ApJ, 529, 338

Thoul, A. A., Bahcall, J. N., \& Loeb, A. 1994, ApJ, 421, 828 